\title{
Added Value of Spectroscopy to Perfusion MRI in the Differential Diagnostic Performance of Common Malignant Brain Tumors
}

\author{
(D) A. Vallée, (D) C. Guillevin, (D) M. Wager, (D) V. Delwail, (DR. Guillevin, and DJ.-N. Vallée
}

\begin{abstract}
BACKGROUND AND PURPOSE: Perfusion and spectroscopic MR imaging provide noninvasive physiologic and metabolic characterization of tissues, which can help in differentiating brain tumors. We investigated the diagnostic role of perfusion and spectroscopic MR imaging using individual and combined classifiers of these modalities and assessed the added performance value that spectroscopy can provide to perfusion using optimal combined classifiers that have the highest differential diagnostic performance to discriminate lymphomas, glioblastomas, and metastases.
\end{abstract}

MATERIALS AND METHODS: From January 2013 to January 2016, fifty-five consecutive patients with histopathologically proved lymphomas, glioblastomas, and metastases were included after undergoing MR imaging. The perfusion parameters (maximum relative CBV, maximum percentage of signal intensity recovery) and spectroscopic concentration ratios (lactate/ $\mathrm{Cr}$, $\mathrm{Cho} / \mathrm{NAA}, \mathrm{Cho} / \mathrm{Cr}$, and lipids/ $\mathrm{Cr}$ ) were analyzed individually and in optimal combinations. Differences among tumor groups, differential diagnostic performance, and differences in discriminatory performance of models with quantification of the added performance value of spectroscopy to perfusion were tested using 1-way ANOVA models, receiver operating characteristic analysis, and comparisons between receiver operating characteristic analysis curves using a bivariate $\chi^{2}$, respectively.

RESULTS: The highest differential diagnostic performance was obtained with the following combined classifiers: maximum percentage of signal intensity recovery-Cho/NAA to discriminate lymphomas from glioblastomas and metastases, significantly increasing the sensitivity from $82.1 \%$ to $95.7 \%$; relative CBV-Cho/NAA to discriminate glioblastomas from lymphomas and metastases, significantly increasing the specificity from $92.7 \%$ to $100 \%$; and maximum percentage of signal intensity recovery-lactate/ $\mathrm{Cr}$ and maximum percentage of signal intensity recovery-Cho/Cr to discriminate metastases from lymphomas and glioblastomas, significantly increasing the specificity from $83.3 \%$ to $97.0 \%$ and $100 \%$, respectively.

CONCLUSIONS: Spectroscopy yielded an added performance value to perfusion using optimal combined classifiers of these modalities, significantly increasing the differential diagnostic performances for these common brain tumors.

ABBREVIATIONS: $\mathrm{AUC}=$ area under the ROC curve; $\triangle \mathrm{R} 2^{*}=$ relaxation rate; $\mathrm{GBM}=$ high-grade gliomas (glioblastomas); Lac = lactate; Lip = lipids; $\mathrm{max}=$ maximum; $\min =$ minimum; PCNSL = primary central nervous system lymphoma; PRESS = point-resolved spectroscopic sequence; $P S R=$ percentage of signal intensity recovery; $\mathrm{rCBV}=$ relative cerebral blood volume; $\mathrm{ROC}=$ receiver operating characteristic

$P$ rimary central nervous system lymphomas (PCNSLs), glioblastomas (GBMs), and metastases are the most commonly identified brain tumors in adults. Management of these malig-

Received September 6, 2017; accepted after revision May 1, 2018.

From the Délégation à la Recherche Clinique et à l'innovation (A.V.), Hôpital Foch, 92150 Suresnes, France; DACTIM-MIS, UMR CNRS 7348 (A.V., C.G., R.G., J.-N.V.), Laboratory of Mathematics and Applications (LMA), University of Poitiers, 86000 Poitiers, France; Institut National de la Santé et de la Recherche Médicale (INSERM) U-1084 (M.W.), Experimental and Clinical Neurosciences Laboratory, University of Poitiers, 86000 Poitiers, France; Departments of Radiology (C.G., R.G.), Neurosurgery (M.W.), and Haematology (V.D.), Poitiers University Hospital, University of Poitiers, 86000 Poitiers, France; and Department of Diagnostic and Interventional Neuroradiology (J.-N.V.), Amiens University Hospital, University Picardie Jules Verne of Amiens, 80054 Amiens, France. nancies can differ substantially depending on lesion type; however, their preoperative characterization and differentiation can be challenging due to the overlapping of their imaging characteristics.

Conventional MR imaging is very limited in making the distinction. Contrast enhancement on T1-weighted images is dependent on blood-brain barrier disruption and neovascularity regardless of the pathologies. FLAIR MR imaging can depict a large

Please address correspondence to J.-N. Vallée, MD, PhD, Department of Diagnostic and Interventional Neuroradiology, Amiens University Hospital, University Picardie Jules Verne of Amiens (UPJV), 80054 Amiens, France; UMR CNRS 7348, Laboratory of Mathematics and Applications, University of Poitiers, Poitiers, France; e-mail: valleejn@gmail.com

http://dx.doi.org/10.3174/ajnr.A5725 
fraction of the tumor but lacks specificity. ${ }^{1}$ Advanced MR imaging modalities such as perfusion-weighted imaging and proton MR spectroscopy ( ${ }^{1} \mathrm{H}-\mathrm{MR}$ spectroscopy) provide a physiologic and metabolic noninvasive characterization of brain tumors, which can help in the differentiation of these lesions. ${ }^{2,3}$ According to a recent meta-analysis, the apparent diffusion coefficient measured in diffusion MR imaging, in particular $\mathrm{ADC}_{\text {mean }}$, correlated with glioma cellularity, but too weakly with lymphoma to be used as a biomarker of cellularity in this entity and with no evident data for metastases provided. ${ }^{4}$

The histopathologic differences in the tumor capillary structure are the origin of the variability in perfusion MR imaging, which may provide the basis for a possible differential diagnosis. PWI provides hemodynamic parameter measurements such as the relative cerebral blood volume ( $\mathrm{rCBV}$ ) as an index of microvascularity and neoangiogenesis correlated with the aggressiveness and malignancy of tumors, and the percentage of signal intensity recovery (PSR) as an index of the tissue capillary permeability. ${ }^{3,5}$ rCBV in PCNSLs is considerably lower than in GBMs and metastases; however, there is an overlap in rCBV values with regard to GBMs and metastases. ${ }^{3,6,7}$ Tumor rCBV measurements reflect gross tumor blood volume but do not provide any information regarding capillary permeability. ${ }^{8}$ Mangla et $\mathrm{al}^{3}$ reported that PSR appears to be a useful parameter to differentiate PCNSLs from GBMs and metastases, or metastases from PCNSLs and GBMs, but not GBMs from PCNSLs and metastases.

${ }^{1} \mathrm{H}-\mathrm{MR}$ spectroscopy allows in vivo detection and characterization of brain metabolites, such as choline, a cellular membrane turnover marker involved in the metabolism of the phospholipid membrane structure (phosphatidylcholine and sphingomyelin) reflecting an increased need for membrane renewal due to alteration/destruction or membrane proliferation; $N$-acetylaspartate, a quantitative neuronal viability marker, its decrease reflecting an indirect measure of neuronal loss or degradation; creatine, a marker of intracellular energetic metabolism; lactate (Lac), usually undetectable by MR imaging in the healthy brain, its presence reflecting a product of anaerobic glycolysis; mobile lipids (Lip), absent in the normal brain, their concentration indicating the extent of necrosis and apoptosis. Changes in these brain metabolites often precede the structural abnormalities of the tumor. By means of characteristic changes in the metabolite profile in certain tumors compared with the normal CNS profile, ${ }^{1} \mathrm{H}$-MR spectroscopy has the potential to provide a biochemical differential diagnosis. Thus, ${ }^{1} \mathrm{H}-\mathrm{MR}$ spectroscopy could yield added value to perfusion MR imaging, increasing the reliability of the preoperative differentiation of PCNSLs, GBMs, and metastases.

We investigated the usefulness of the diagnostic role of perfusion and spectroscopic MR imaging using individual and combined classifiers of these modalities based on physiologic and biometabolic differences of tumors. Then, we assessed and quantified the added performance value that spectroscopy can provide to perfusion using optimal combined classifiers that have the highest differential diagnostic performance to discriminate lymphomas, glioblastomas, and metastases.

\section{MATERIALS AND METHODS \\ Patients}

Sixty-one consecutive patients with histopathologically proved PCNSL, GBM, or solitary metastases were evaluated from January 2013 to January 2016, after obtaining prior approval of the institutional review board (CHU Poitiers, France) and informed consent of each patient. Six patients were excluded because of artifacts on perfusion data (3 patients), uninterpretable spectral data (2 patients), and an immunocompromised condition (1 patient). Thus, we included 55 patients ( 23 women, 32 men; mean age, $63.8 \pm 13.7$ years); 28 previously untreated immunocompetent patients with PCNSL, 14 with GBM, and 13 with solitary metastases ( 5 originating from lung; 3 , from breast; 2 , from thyroid; 2 , from melanoma; 1 , from the esophagus) were analyzed.

\section{Imaging Protocol}

Conventional MR Imaging. All patients underwent an MR imaging examination according to the same protocol using a wholebody system (Verio 3T; Siemens, Erlangen, Germany) and the following features: sagittal 3D-FLAIR (TR/TE/TI $=5000 / 402 /$ $1800 \mathrm{~ms}, \mathrm{FOV}=260 \mathrm{~mm}$, matrix $=156 \times 128)$, axial 3D-T1 postcontrast $\left(\mathrm{TR} / \mathrm{TE} / \mathrm{TI}=1900 / 2.93 / 900 \mathrm{~ms}\right.$, flip angle $=9^{\circ}$, $\mathrm{FOV}=255 \mathrm{~mm}$, matrix $=256 \times 256)$, and axial postcontrast T1-weighted FSE imaging $(\mathrm{TR} / \mathrm{TE}=308 / 2.48 \mathrm{~ms}, \mathrm{FOV}=220$ mm, matrix $=272 \times 352)$.

PWI. Dynamic-susceptibility perfusion contrast-enhanced $\mathrm{T} 2{ }^{*}$ weighted gradient-echo echo-planar images $(\mathrm{TR} / \mathrm{TE}=1980 / 30$ $\mathrm{ms}$, 4.0-mm thick sections, $0.8-\mathrm{mm}$ gap, FOV $=220 \mathrm{~mm}$, matrix $=128 \times 128$, flip angle $=90^{\circ}$, phases $\left.=75\right)$ were acquired during the first pass of a standard-dose $(0.1 \mathrm{mmol} / \mathrm{Kg})$ bolus of gadoteric acid at $0.5 \mathrm{mmol} / \mathrm{mL}$. Contrast material was injected at a rate of $6 \mathrm{~mL} / \mathrm{s}$ for all patients, with a 10-second delay. From 7 to 12 sections were selected on T2 FLAIR-weighted images, depending on the volume of the tumor.

'H-MR Spectroscopy. All the spectroscopic data were obtained after intravenous administration of gadoteric acid using a pointresolved spectroscopic sequence (PRESS: TR $=1500 \mathrm{~ms} / \mathrm{TEs}=$ 35/135 ms, 156 scans). CBV cartographies derived from PWI processing were used to position the excitation box sized from 10 to $15 \mathrm{~mm}$ in all 3 dimensions, depending on the volume of the lesion. Voxels were positioned within the ROI that allowed the maximal value of rCBV. Another equally sized voxel was symmetrically positioned in the healthy contralateral parenchyma. We selected the VOI to include the lesion and areas of healthy contralateral brain parenchyma while avoiding the scalp, skull, and sinuses.

\section{Postprocessing}

rCBV measurements from DSC MR imaging data were performed using syngo.via software (Siemens). During the first pass of a bolus of contrast agent, $\mathrm{T} 2^{*}$-weighted signal intensity decreased. The change in the relaxation rate $\left(\Delta \mathrm{R} 2^{*}, \mathrm{ie}\right.$, the change in the reciprocal of $\left.\mathrm{T} 2^{*}\right)$ can be calculated from the signal intensity as follows: $\Delta \mathrm{R} 2^{*}(t)=\{-\ln [S(t) / S o]\} / \mathrm{TE}$, where $S(t)$ is the signal intensity at time $t$, and $S o$, the unenhanced signal intensity. $\Delta \mathrm{R} 2^{*}$ is proportional to the concentration of contrast agent in the tissue, and CBV is proportional to the area under the curve of $\Delta \mathrm{R} 2{ }^{\star}(t)$, 
provided there is no recirculation or leakage of contrast agent. In general, these assumptions are violated, but the effects can be reduced by fitting a $\gamma$-variate function to the measured $\Delta \mathrm{R} 2^{*}$ curve. This function approximates the curve that would have been obtained without recirculation or leakage. CBV can then be estimated from the area under the fitted curve rather than from the original data. ROIs of standardized size (4.5-mm radius) were placed in regions of maximal CBV on CBV color overlay maps for targeting and were referenced to the symmetrically contralateral parenchyma (normal white or gray matter) for the calculation of relative maximal $\mathrm{CBV}, \mathrm{rCBV}_{\max }=\mathrm{CBV}_{\text {lesion }} / \mathrm{CBV}_{\text {contralateral }}$. Volume averaging with the blood vessels was carefully avoided, confounding factors in the CBV analysis were minimized, and the size of the ROIs was kept constant. Because the maximally perfused regions of gliomas are suggestive of aggressiveness, ${ }^{3,5,6}$ we analyzed the PWI data from the maximally perfused regions of the tumors by drawing from 5 to 27 ROIs to cover the entire tumor volume, depending on the tumors.

PSR measurements were performed using syngo.via software. ROIs were drawn on the gray-scale perfusion maps overlaid on contrast-enhancing tumor on T1-weighted images. An ROI of $30-40 \mathrm{~mm}^{2}$ was moved within the tumor area to look for the highest and lowest recoveries on $\mathrm{T} 2^{\star}$-weighted signal intensity curves and was selected for maximum and minimum PSR, respectively. For normalization, an ROI of approximately $30-50 \mathrm{~mm}^{2}$ was also placed in the symmetrically contralateral parenchyma, and ratios were obtained. The PSR was calculated as described by Cha et al: ${ }^{7} \mathrm{PSR}=100 \% \times\left(S_{1}-S_{\min }\right) /\left(S_{0}-S_{\min }\right)$, where $S_{1}, S_{0}$, and $S_{\min }$ are postcontrast, precontrast, and minimum $\mathrm{T}^{\star}{ }^{\star}$ weighted signal intensities, respectively.

${ }^{1} \mathrm{H}-\mathrm{MR}$ spectroscopy raw data were analyzed with jMRUI (http://www.jmrui.eu/ $)^{9}$ postprocessing software using the AMARES algorithm ${ }^{10}$ for accurate relative quantification. The data were normalized using the contralateral creatine resonance signal from the symmetric healthy parenchyma. Cho (3.22 ppm), NAA (2.02 ppm), Cr (3.02 ppm), and Lac (1.33 ppm) resonances were assessed at an intermediate TE (135 ms), and free Lip (0.9$1.3 \mathrm{ppm}$ ) resonances were assessed at a short TE (35 ms). Both long TE and short TE spectra were used because the lactate doublet was inverted at 13-ms TE due to the J-coupling, thus allowing the separation and the correct quantification of lactate and lipids.

The structural image-processing tool FSL (http://www. fmrib.ox.ac.uk/fsl) was used to estimate the gray matter, white matter, and CSF content of each voxel and to correct for the partial volume effects on the metabolite data. Brain tissue images were extracted by removing the outer skull and scalp surfaces using the FSL Brain Extraction Tool (http://fsl.fmrib.ox.ac.uk/ fsl/fslwiki/BET). ${ }^{11}$ Finally, the FAST/FIRST tool (https://fsl. fmrib.ox.ac.uk/fsl/fslwiki/FAST; http://fsl.fmrib.ox.ac.uk/fsl/ fslwiki/FIRST) was used to calculate the segmented tissue percentage in the VOI. Coregistration between the spectroscopic VOI and the segmented image was performed with a user-developed Matlab program (MathWorks, Natick, Massachusetts).

\section{Statistical Analysis}

The variables assessed included tumor groups (PCNSLs, GBMs, metastases), maximum perfusion parameters $\left(\mathrm{rCBV}_{\max }\right.$,
$\mathrm{PSR}_{\max }$ ), and spectroscopic concentration ratios (Lac/Cr, Cho/ NAA, Cho/Cr, Lip/Cr). Differences among groups were tested using 1-way ANOVA and the Fisher PLSD post hoc test (Fisher's Protected Least Significant Difference) (SAS 9.1; SAS Institute, Cary, North Carolina).

ROC analysis was performed for individual classifiers of spectroscopy and perfusion regarding their predictive ability to discriminate these tumors. For each classifier, the ability of the logistic regression models to allow discrimination was quantified by the area under the ROC curve (AUC). The maximum Youden index, $J=\max _{c}[S e(c)+S p(c)-1]$, was chosen to determine the optimal decision thresholds $(c)$ for the discrimination.

Next, combined ROC analysis was performed for the classifiers resulting from the different combinations between a spectroscopy classifier and a perfusion classifier using the Logistic Regression Multivariate Effect Likelihood Ratio Tests ${ }^{12}$ (SAS 9.1) to assess the discriminatory performance (AUC) for the differential diagnosis of these tumors. The classifiers used for the combination of classifiers were identified among those previously screened with the ROC analysis for individual classifiers of spectroscopy and perfusion with a level of $P<.05$.

Differences between the AUC values of ROC curves were tested for significance using a bivariate $\chi^{2}$ test (SAS 9.1) to determine the differences among the predictive abilities in discriminatory performance (AUC) of classifiers (individual and combined) for the differential diagnosis of PCNSLs, GBMs, and metastases and to quantify the added performance value of spectroscopy to perfusion.

Mean values were expressed with their SDs. Significance was $P<.05$.

\section{RESULTS \\ Perfusion and Spectroscopy Parameters and Signal Intensity Curves}

Mean values of perfusion parameters $\left(\mathrm{rCBV}, \mathrm{PSR}_{\max }\right.$ ) and spectroscopic concentration ratios (Cho/Cr, Cho/NAA, Lac/Cr, Lip/ $\mathrm{Cr}$ ) in the tumor groups PCNSL, GBM, and metastases are shown in Table 1 and Fig 1; and signal intensity curves and cerebral MR imaging maps with postprocessing, in Fig 2. One-way ANOVA of the mean and SD derived for the perfusion and spectroscopy parameters showed significant differences among the 3 groups of lesions for all parameters studied. The Fisher PSLD test for pairwise comparisons demonstrated, in PCNSLs versus GBMs and metastases, significantly higher values of $\operatorname{PSR}_{\max }(P=.001$, respectively) and lower values of Cho/NAA $(P=.001)$; in GBMs versus PCNSLs and metastases, significantly higher values of $\operatorname{rCBV}(P=.001$, respectively) and Cho/NAA $(P=.001)$; and in metastases versus PCNSLs and GBMs, significantly lower values of $\operatorname{PSR}_{\max }(P=.001, .02$, respectively $)$, Cho/Cr $(P=.002, .03$, respectively), and $\operatorname{Lac} / \mathrm{Cr}(P=.001, .01$, respectively $)$ and higher values of $\operatorname{Lip} / \operatorname{Cr}(P=.04, .03$, respectively) (Table 1 and Fig 1$)$.

\section{Accuracy of Individual Classifiers}

The AUCs obtained from ROC curves and decision thresholds for individual classifiers of perfusion and spectroscopy are shown in Table 2. The accuracy of the classifiers Cho/NAA and PSR max $_{\text {was }}$ not significantly different $(\mathrm{AUC}=0.835,0.940, P=.82)$ and was 
Table 1: Mean values of perfusion parameters and spectroscopic concentration ratios in differentiating brain tumors

\begin{tabular}{|c|c|c|c|c|c|c|}
\hline $\begin{array}{c}\text { Oncotype of Lesions/ } \\
P \text { and } F \text { Values }\end{array}$ & rCBV & $\mathrm{PSR}_{\max }$ & $\mathrm{Cho} / \mathrm{Cr}$ & Cho/NAA & $\mathrm{Lac} / \mathrm{Cr}$ & Lip/Cr \\
\hline PCNSL & $2.126 \pm 0.335$ & $106.857 \pm 19.165$ & $3.092 \pm 1.752$ & $2.007 \pm 1.613$ & $2.529 \pm 2.334$ & $3.379 \pm 4.522$ \\
\hline GBM & $3.573 \pm 0.851$ & $74.857 \pm 7.564$ & $2.723 \pm 0.732$ & $3.703 \pm 0.878$ & $2.297 \pm 1.102$ & $2.865 \pm 3.371$ \\
\hline Metastases & $2.445 \pm 0.805$ & $60.545 \pm 7.634$ & $1.568 \pm 0.282$ & - & $0.186 \pm 0.336$ & $6.346 \pm 3.533$ \\
\hline$P($ ANOVA $)$ & $<.001^{\mathrm{a}}$ & $<.001^{\mathrm{a}}$ & $.009^{a}$ & $.001^{\mathrm{a}}$ & $.003^{a}$ & .07 \\
\hline$F($ ANOVA) & 26.083 & 45.876 & 5.208 & 13.024 & 6.680 & 2.712 \\
\hline P PCNSL vs GBM & $<.001^{\mathrm{a}}$ & $<.001^{\mathrm{a}}$ & .40 & $.001^{\mathrm{a}}$ & .73 & .70 \\
\hline P PCNSL vs metastases & .15 & $<.001^{\mathrm{a}}$ & $.002^{\mathrm{a}}$ & & $.001^{\mathrm{a}}$ & $.04^{\mathrm{a}}$ \\
\hline$P$ GBM vs metastases & $<.001^{\mathrm{a}}$ & $.02^{\mathrm{a}}$ & $.03^{\mathrm{a}}$ & & $.001^{\mathrm{a}}$ & $.03^{\mathrm{a}}$ \\
\hline
\end{tabular}

Note:- - NAA was undetectable at $2.02 \mathrm{ppm}$ in metastases.

a Significant.
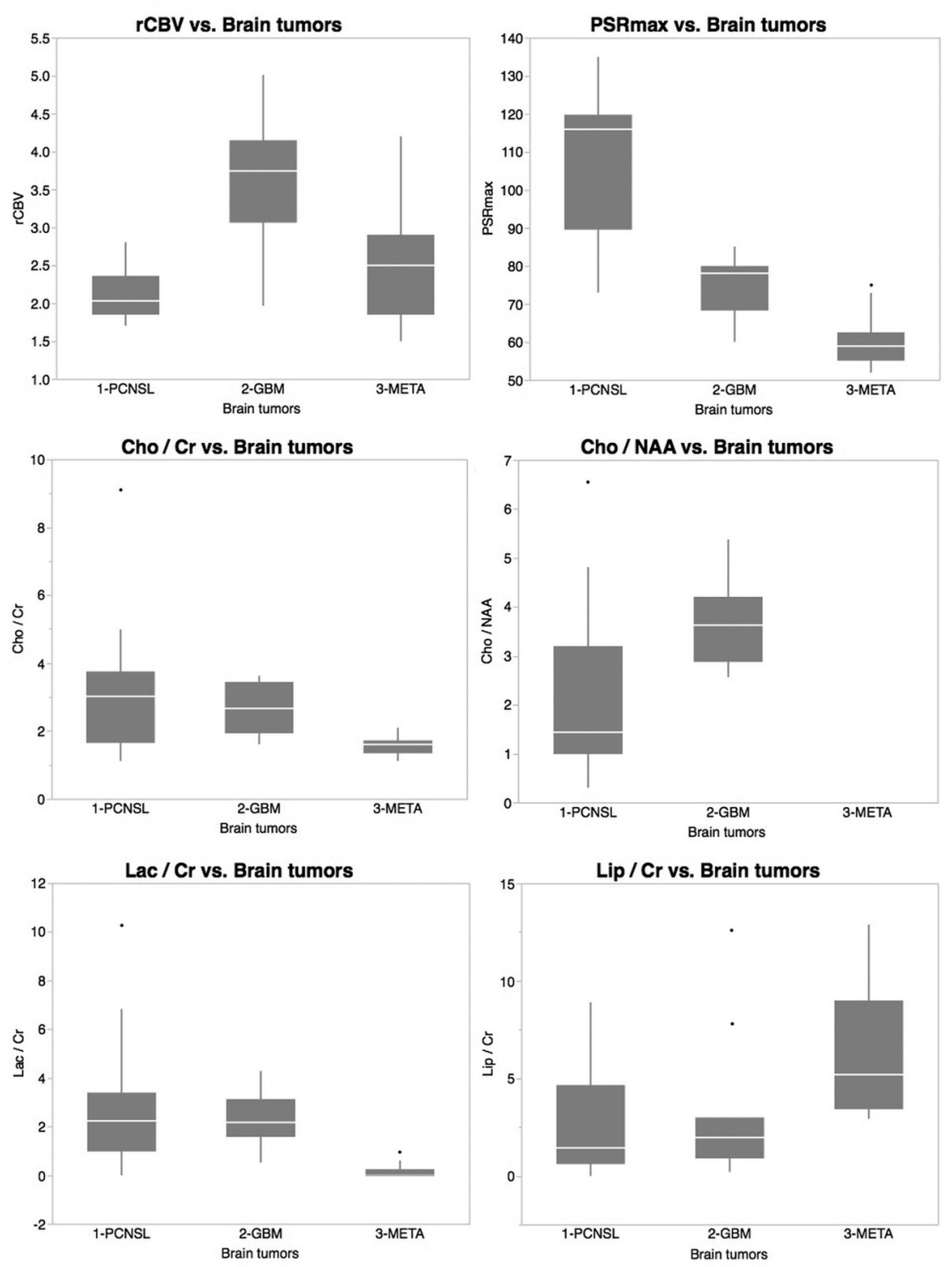

FIG 1. Boxplots of perfusion parameters and spectroscopic concentration ratios from MR imaging in differentiating brain tumors.

higher than that of other MR imaging classifiers $(P<.05)$ to differentiate PCNSLs from GBMs and metastases. The accuracy of the classifiers Cho/NAA and rCBV was not significantly different $(\mathrm{AUC}=0.835,0.911, P=.41)$ and was higher than that of other MR imaging classifiers $(P<.05)$ to differentiate GBMs from PCNSLs and metastases. The accuracy of the classifiers Lac/Cr,
Cho/Cr, and $\mathrm{PSR}_{\max }$ was not significantly different (AUC $=0.957,0.868$, $0.969, P=.11, .69, .16)$ and was higher than that of other MR imaging classifiers $(P<.05)$ to differentiate metastases from PCNSLs and GBMs (Fig 3).

\section{Accuracy of Combined Classifiers}

Comparisons between AUCs obtained from ROCs for the individual and combined classifiers showed that the combined classifier PSR $_{\max }-\mathrm{Cho} / \mathrm{NAA}$ had the highest differential diagnostic performance to discriminate PCNSLs from GBMs and metastases with significantly higher accuracy than that of the best individual classifiers, $\mathrm{PSR}_{\max }$ and Cho/ NAA (AUC $=0.988$ versus 0.940 and 0.835; $P<.001$ and .02 , respectively), a sensitivity of $95.7 \%$, and a specificity of $100 \%$, thus significantly increasing the sensitivity of perfusion $(82.1 \%)$ and spectroscopy $(73.9 \%)$ to $95.7 \%$. In the differentiation of GBMs and PCNSLs and metastases, the combined classifier rCBV-Cho/NAA had the highest differential diagnostic performance with significantly higher accuracy than that of the best individual classifiers, $\mathrm{rCBV}$ and Cho/NAA $(\mathrm{AUC}=0.960$ versus 0.911 and $0.835 ; P=.001$ and .005 , respectively), a sensitivity of $85.7 \%$, and a specificity of $100 \%$, thus significantly increasing the specificity of perfusion $(92.7 \%)$ and spectroscopy $(73.9 \%)$ to $100 \%$. In the differentiation of metastases versus PCNSLs and GBMs, the combined classifiers ( $\mathrm{PSR}_{\max }-\mathrm{Lac} / \mathrm{Cr}$ ) and $\left(\mathrm{PSR}_{\max }-\mathrm{Cho} / \mathrm{Cr}\right.$ ) had the highest differential diagnostic performances with accuracies not significantly different $(\mathrm{AUC}=0.988$ and $1 ; P=.89$ ), but significantly higher than those of the best individual classifiers $\mathrm{PSR}_{\max }, \mathrm{Lac} / \mathrm{Cr}$, and Cho/Cr $(\mathrm{AUC}=0.969$, 0.957 and $0.868 ; P=.001, .001, .04$ and $.001, .001, .02$, respectively), a sensitivity of $100 \%$, respectively, and specificities of 

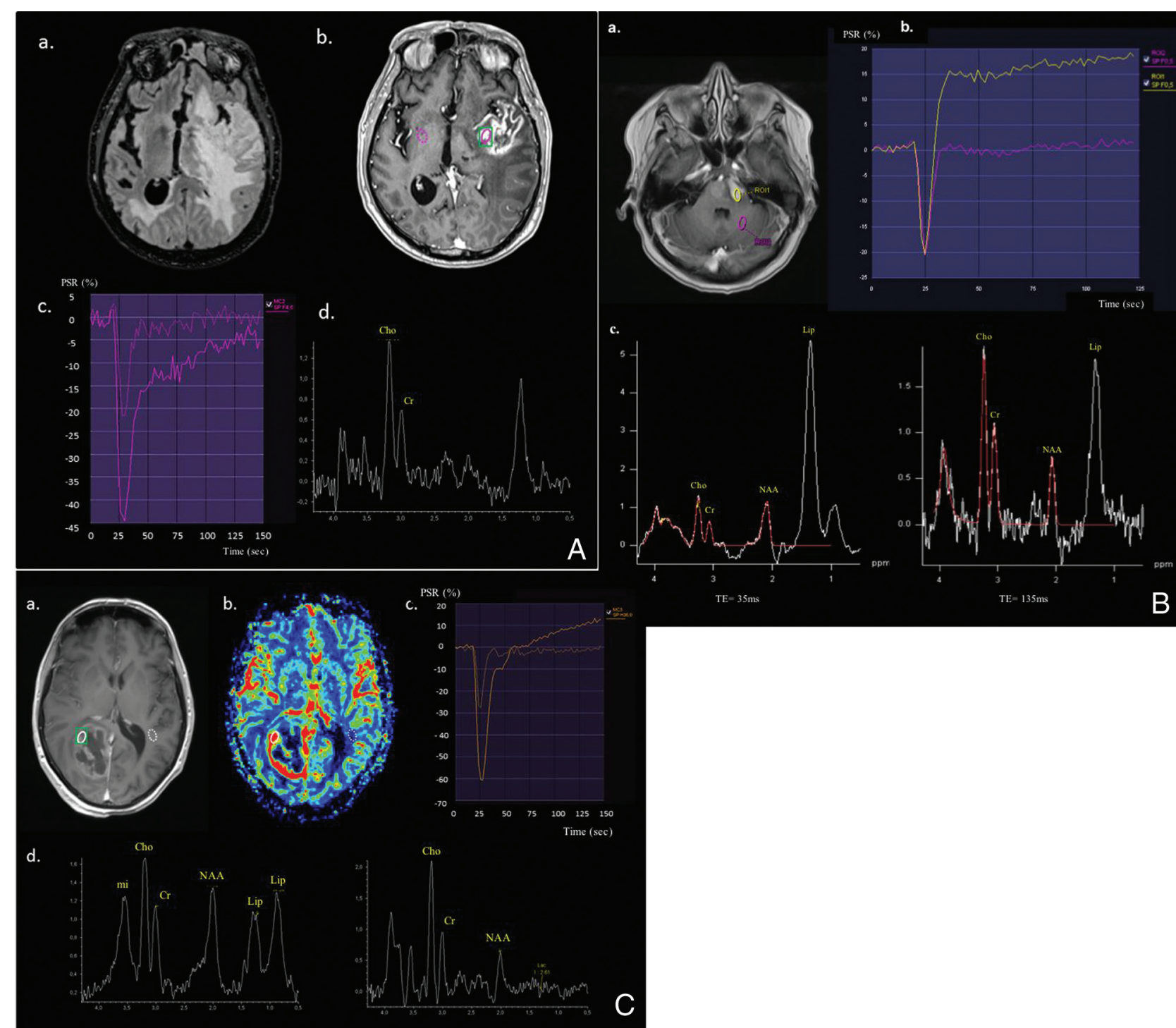

FIG 2. Cerebral MR imaging with postprocessing using syngo.via software shows the following: $A$, A 77-year-old man with histologically proved left insula metastasis from lung cancer who has extensive peritumoral edema on the T2-weighted FLAIR image (a), a heterogeneous contrast enhancement with necrosis on the postcontrast Tl-weighted image (b), $\mathrm{PSR}_{\max }$ at $65 \%$ with $\mathrm{rCBV}_{\max }$ at 2.5 on PWI $\gamma$ function (c), and the Cho/Cr ratio at 1.8 with the resonance of free lipids still visible at TE $=135 \mathrm{~ms}$ on ${ }^{1} \mathrm{H}$-MR PRESS spectra (d). $B, A$. 56-year-old man with histologically proved PCNSL within the pons who has a homogeneous hyperintense lesion on postcontrast T1-weighted image (a), $\mathrm{PSR}_{\max }$ at $125 \%$ with no increase in $\mathrm{rCBV}_{\max }$ on PWI $\gamma$ function (b), and a strong resonance of free lipids at a short TE $=35 \mathrm{~ms}$, as well as at a long TE $=135 \mathrm{~ms}$ on ${ }^{1} \mathrm{H}-\mathrm{MR}$ PRESS spectra. Note also a strong increase of Cho/NAA and Cho/ $\mathrm{Cr}$ at TE $=135 \mathrm{~ms}$ (c). C, A 52-year-old woman with histologically proved right occipital glioblastoma who has a heterogeneous necrotic lesion on the postcontrast T1-weighted image (a), with a ring of hyperperfusion (b), PSR max $_{\text {at }}$ $80 \%$ with rCBV $\max$ at 4.1 on the PWI $\gamma$ function (c), and a strong resonance of free lipids at TE $=35$ ms and lactate with ratios of Cho/Cr at 3.1 and Cho/NAA at 3.5 on ${ }^{~} \mathrm{H}-\mathrm{MR}$ PRESS spectra (d).

$97.0 \%$ and $100 \%$, respectively, thus significantly increasing the specificity of perfusion $(83.3 \%)$ and spectroscopy $(81.8 \%)$ to 97.0\% using the classifier $\mathrm{PSR}_{\max }-\mathrm{Lac} / \mathrm{Cr}$ and $100 \%$ using the classifier $\mathrm{PSR}_{\max }-\mathrm{Cho} / \mathrm{Cr}$ (Table 2 and Fig 3 ).

\section{DISCUSSION}

In our study, we have given special attention to the clinical application of our results by the determination of MR imaging classifiers that have the maximum differential diagnostic performance to discriminate brain tumor pathologies, which is more useful and closer to reasoning for the decision-making in daily clinical practice.

\section{Differentiation of PCNSLs from GBMs and Metastases}

Our study showed that the increased capillary permeability $\left(\mathrm{PSR}_{\max }\right.$ ) was significantly greater in PCNSLs than that in GBMs and metastases and is concordant with several studies. ${ }^{3,7,8}$ These findings suggest that contrast material leakage into the interstitial space is more pronounced in PCNSLs than in GBMs and metastases. ${ }^{1,13}$ Mangla et $\mathrm{al}^{3}$ and Paulson and Schmainda ${ }^{14}$ reported that the intensity of signal for DSC imaging is the combined result of the $\mathrm{T} 2{ }^{*}$ and $\mathrm{T} 1$ shortening effect from the accumulated contrast agent in the interstitial space. The $\mathrm{T} 2^{\star}$ effects cause lower signal intensity recovery, while the $\mathrm{T} 1$ effects lead to higher signal intensity recovery. The signal intensity increases and even exceeds 
baseline when the T1 shortening effect of the accumulation of contrast material in the interstitial space dominates the $\mathrm{T} 2{ }^{*}$ effect. Xing et $\mathrm{al}^{8}{ }^{8}$ reported that in PCNSLs, the contrast accumulation might be slow and $\mathrm{T} 1$ and $\mathrm{T} 2{ }^{*}$ shortening effects are not apparent during the first pass. Only after the first pass does the T1 shortening effect overwhelm the $\mathrm{T} 2^{\star}$ shortening effect, resulting in a higher PSR. In most GBMs and metastases of high vessel density, the $2_{2}^{*}$ effect from rapid and abundant accumulation of contrast agent in the interstitial space is considerably greater than the T1 shortening effect during and even after the first-pass period, leading to a lower PSR.

The markedly increased PSR in PCNSLs has so far not been

Table 2: AUC from the ROC for individual and combined classifiers and decision thresholds, to discriminate brain tumors

\begin{tabular}{|c|c|c|c|c|c|}
\hline & AUC & $P$ Value & Thresholds & Sensitivity & Specificity \\
\hline \multicolumn{6}{|c|}{ PCNSL vs GBM, metastases } \\
\hline $\mathrm{PSR}_{\max }$ & 0.940 & $<.001^{\mathrm{a}}$ & $\geq 87.00$ & 82.1 & 100.0 \\
\hline Cho/NAA & 0.835 & $<.001^{\mathrm{a}}$ & $\leq 2.07$ & 73.9 & 100.0 \\
\hline rCBV & 0.789 & $<.001^{\mathrm{a}}$ & $\leq 2.59$ & 92.9 & 63.0 \\
\hline $\mathrm{Lac} / \mathrm{Cr}$ & 0.728 & $.006^{\mathrm{a}}$ & $\geq 0.87$ & 87.0 & 56.5 \\
\hline $\mathrm{Cho} / \mathrm{Cr}$ & 0.665 & $.01^{\mathrm{a}}$ & $\geq 2.84$ & 56.5 & 77.8 \\
\hline $\mathrm{Lip} / \mathrm{Cr}$ & 0.638 & .32 & & & \\
\hline $\mathrm{PSR}_{\max }-\mathrm{Cho} / \mathrm{NAA}$ & 0.988 & $<.001^{\mathrm{a}}$ & & 95.7 & 100.0 \\
\hline \multicolumn{6}{|c|}{ GBM vs PCNSL, metastases } \\
\hline rCBV & 0.911 & $<.001^{\mathrm{a}}$ & $\geq 2.86$ & 85.7 & 92.7 \\
\hline Cho/NAA & 0.835 & $<.001^{\mathrm{a}}$ & $\geq 2.57$ & 100.0 & 73.9 \\
\hline $\mathrm{Lac} / \mathrm{Cr}$ & 0.710 & .40 & & & \\
\hline $\mathrm{Cho} / \mathrm{Cr}$ & 0.648 & .68 & & & \\
\hline $\mathrm{PSR}_{\max }$ & 0.633 & .18 & & & \\
\hline $\mathrm{Lip} / \mathrm{Cr}$ & 0.629 & .19 & & & \\
\hline rCBV-Cho/NAA & 0.960 & $<.001^{\mathrm{a}}$ & & 85.7 & 100.0 \\
\hline \multicolumn{6}{|c|}{ Metastases vs PCNSL, GBM } \\
\hline $\mathrm{PSR}_{\max }$ & 0.969 & $<.001^{\mathrm{a}}$ & $\leq 75.00$ & 100.0 & 83.3 \\
\hline $\mathrm{Lac} / \mathrm{Cr}$ & 0.957 & $<.001^{\mathrm{a}}$ & $\leq 0.95$ & 100.0 & 81.8 \\
\hline Cho/Cr & 0.868 & $<.001^{\mathrm{a}}$ & $\leq 1.80$ & 92.3 & 81.1 \\
\hline $\mathrm{Lip} / \mathrm{Cr}$ & 0.813 & $.02^{\mathrm{a}}$ & $\geq 2.90$ & 100.0 & 64.9 \\
\hline rCBV & 0.532 & .51 & & & \\
\hline $\mathrm{PSR}_{\max }-\mathrm{Lac} / \mathrm{Cr}$ & 0.988 & $<.001^{\mathrm{a}}$ & & 100.00 & 97.0 \\
\hline $\mathrm{PSR}_{\max }-\mathrm{Cho} / \mathrm{Cr}$ & 1.000 & $<.001^{\mathrm{a}}$ & & 100.00 & 100.0 \\
\hline
\end{tabular}

clearly explained..$^{9,14-16}$ In contrast to GBMs and metastases, the tumor hypercellularity according to the angiocentric growth pattern, in which the PCNSL cells tend to cluster around pre-existing brain vessels and form multiple thick layers around the host vessels associated with widening of the perivascular space with smaller extravascular spaces and absence of neoangiogenesis, might explain the faster signal recovery. ${ }^{17}$

PCNSLs demonstrated significantly lower rCBV values and higher PSR values compared with GBMs and metastases, consistent with some authors. ${ }^{1-3,13,18,19}$ These differences have been attributed to the lack of neoangiogenesis and the angiocentric growth pattern in PCNSLs, resulting in lower microvessel density and higher vascular permeability, whereas GBMs or brain metastases are characterized by tumor microvasculature and angiogenesis. $3,13,20$

However, some authors ${ }^{3,8,19}$ have demonstrated that PSR was a significantly more accurate predictor than rCBV in differentiating PCNSLs from GBMs and metastases, which is in agreement with our findings, with an accuracy of 0.940 and 0.789 , respectively.

In our study, the Cho/Cr ratio increased in both PCNSLs and GBMs without a significant difference between the 2 groups. However, the Cho/NAA ratio increased both in PCNSLs and GBMs but with significantly lower values in PCNSLs than in GBMs. The NAA/Cr ratio decreased less in PCNSLs than in GBMs. Thus, neuronal structures were less affected by PCNSLs than GBMs.

A trend toward higher Lip/Cr levels in PCNSLs compared with GBMs proved to be statistically nonsignificant. Some authors $^{21-23}$ stated that markedly elevated

a Significant.

Lymphomas vs (glioblastomas-metastases)

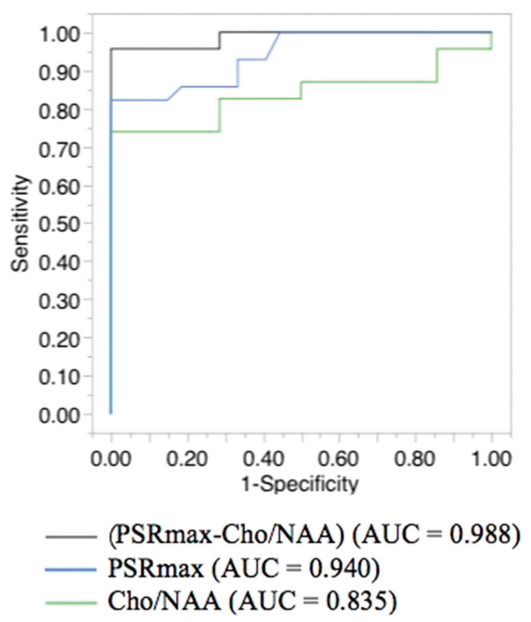

Glioblastomas vs (lymphomas-metastases)

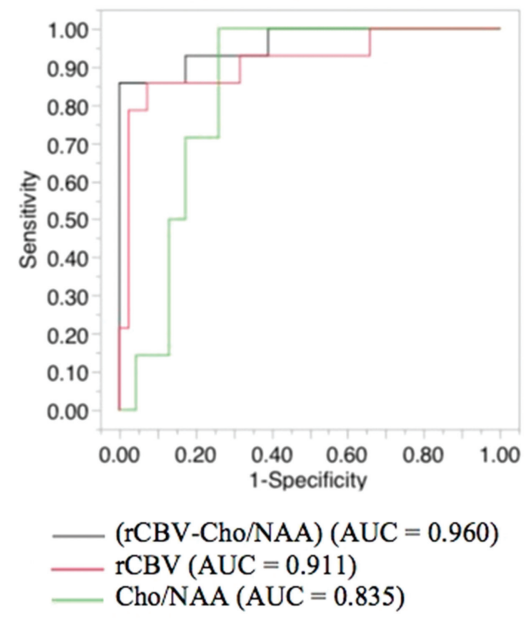

Metastases vs (lymphomas-glioblastomas)

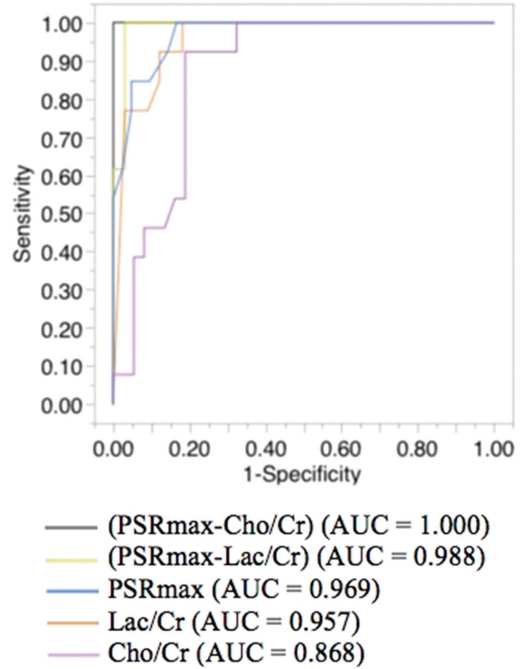

FIG 3. Differential diagnostic performances and the differences in discriminatory performance of classifiers of spectroscopy and perfusion to discriminate lymphomas, glioblastomas, and metastases. 
lipid and choline in PCNSLs might be helpful in differentiating them from GBMs. Although the origin of the lipid is still unclear, an increase in lipid resonance is usually associated with necrosis and membrane breakdown as a signature of cell death. ${ }^{24}$ However, a lipiddominated spectrum found in PCNSLs is not macroscopically necrotic $^{23}$ and may result from the contribution of numerous macrophages and the increased turnover of the membrane components in transformed lymphoid cells rather than from necrosis. ${ }^{25}$

Thus, our study demonstrated that the combined assessment of capillary permeability and cellular membrane turnover by proliferating cells relative to the viability of neuronal structures using the combined classifier $\mathrm{PSR}_{\max }-\mathrm{Cho} / \mathrm{NAA}$ was the underlying physiologic and metabolic characteristic that provides the best differential diagnostic performance to discriminate PCNSLs from GBMs and metastases.

\section{Differentiation of GBMs from PCNSLs and Metastases}

Our study showed that rCBV increased in GBMs with significantly higher values than in PCNSLs and metastases. The ultrastructure of $\mathrm{GBMs}^{2,6,26}$ is characterized by tumor microvasculature and extensive angiogenesis, including glomeruloid capillaries, simple vascular hyperplasia, delicate neocapillaries, and blood-brain barrier disruption, which may explain an increase in rCBV within the tumor compared with normal white or gray matter. ${ }^{27}$ Increased rCBV, considerably higher in GBMs, correlates with the neoangiogenesis of malignant gliomas ${ }^{3,5}$ and has been used in the grading of gliomas. ${ }^{6}$

The Cho/NAA ratio increased in GBMs with significantly higher values than in PCNSLs. Thus, in GBMs, the decreased neuronal and axonal viability was significantly greater than that in PCNSLs because the Cho/Cr ratio was not significantly different between PCNSLs and GBMs, indicating that neuronal loss or degradation was significantly higher in GBMs than in PCNSLs.

PSR was not a sufficiently accurate classifier to exploit the difference in tumor capillary permeability between GBMs and the other common brain tumors such as PCNSLs and metastases; this finding is in agreement with those reported by Mangla et al. ${ }^{3}$ This may be explained by the disparity in capillary permeability characteristics between PCNSLs and metastases, with higher PSR values in PCNSLs and lower PSR values in metastases, compared with GBMs, and the difference in PSR values between PCNSLs and metastases being significantly different.

Thus, combined assessment of microvascularity and angiogenesis and cellular membrane turnover reflecting cell proliferation relative to neuronal viability using the combined classifier rCBV-Cho/NAA was the underlying physiologic and metabolic characteristic that provided the best differential diagnostic performance to discriminate GBMs from PCNSLs and metastases.

\section{Differentiation of Metastases from PCNSLs and GBMs}

Our study showed that PSR ${ }_{\max }$ increased in metastases, but with significantly lower values than those in PCNSLs and GBMs, a finding consistent with those in some reports. ${ }^{7}$ The capillary ultrastructure of metastases, characterized by a vascular structure similar to that of the primary tumor, absence of similarity to normal brain capillaries, prominent capillary fenestrations, and complete lack of BBB components may result in a higher permeability and render metastases far more susceptible to leakage. ${ }^{26}$ The T2* effects from markedly decreased intravascular contrast agent concentration and an increased volume of distribution result in an increase in effective compartment size during the first pass, and it, in turn, alters the signal intensity in a complex way, leading to lower PSR in metastases. ${ }^{3}$

The Lac/Cr ratio increased in metastases but with significantly lower values than those in PCNSLs and GBMs. Lactate detection precedes cellular injury and reflects pathologic conditions associated with an increased energy demand: impaired cellular capability for oxidative phosphorylation, which indicates an increase in anaerobic metabolism; and the presence of ischemic processes and macrophage invasion. ${ }^{28}$ In our study, these pathologic conditions in metastases appeared significantly less important than those in PCNSLs and GBMs. J-coupling was used between TEs of 35 and $135 \mathrm{~ms}$ to extract the lactate resonance by subtraction between the overall co-resonance of lactate + free lipids at TE $=$ $35 \mathrm{~ms}$ and the reversed lactate resonance with respect to the baseline at $\mathrm{TE}=135 \mathrm{~ms}$, thus allowing the separation and the correct quantification of lactate and lipids.

The Cho/Cr ratio increased in metastases but with significantly lower values than those in PCNSLs and GBMs. In tumors, Cho levels correlated with the degree of malignancy, reflective of cellularity. ${ }^{29}$ Increased phosphocholine turnover due to membrane biosynthesis by proliferating cells in metastases was significantly less than that in PCNSLs and GBMs. However, some studies reported that the $\mathrm{Cho} / \mathrm{Cr}$ ratio alone is not reliable in differentiating brain metastases from GBMs. ${ }^{30}$ The prevailing view is that an elevated choline peak is a surrogate marker of increased cell membrane turnover caused by tumor growth or normal cell destruction; however, an alternative view suggests that the choline signal may, in part, be elevated because of increased production through phospholipase upregulation. ${ }^{31}$

Free lipids increased in metastases with significantly higher values than in PCNSLs and GBMs. This finding is consistent with those in previous studies that demonstrated a significant correlation between lipid levels and the amount of necrosis in GBMs and metastases irrespective of their primary origin. ${ }^{32-34}$ Sjøbakk et $\mathrm{al}^{34}$ also confirmed the cytoplasmic origin of MR imaging-visible lipid signals in metastases because a cellular response to stressful stimuli from the tumor microenvironment can result in accumulation of neutral lipids in cytoplasm. MR imaging-visible lipid signals in non-necrotic brain tumor biopsies were mobile lipid droplets of cytoplasmic origin. ${ }^{34,35}$ Furthermore, apoptosis and hypoxic cells have been reported to contribute to elevated spectral lipid signals. ${ }^{36}$

In our study, the Lip/Cr ratio was a discriminant classifier but with a moderate accuracy of 0.813 and a low specificity of $64.9 \%$ to discriminate metastases from PCNSLs and GBMs. While brain metastases demonstrated an elevated lipid peak, ${ }^{20,27}$ this peak was not reliable to discriminate brain metastases from GBMs, which may also be necrotic. ${ }^{37}$

rCBV was not a sufficiently accurate classifier to exploit the difference in tumor angiogenesis between metastases and the other common brain tumors such as PCNSLs and GBMs; this finding is in agreement with those reported by Mangla et al. ${ }^{3}$ This may be explained by the disparity in angiogenesis characteristics between PCNSLs and GBMs, with lower rCBV values in PCNSLs 
and higher rCBV values in GBMs compared with metastases and the difference in rCBV values between GBMs and PCNSLs being significantly different. With regard to GBMs and some hematogenous brain metastases, there may be an overlap in rCBV values because both have high rCBVs. ${ }^{7}$ Several prior studies have reported that rCBV may not be helpful for discrimination of GBMs and brain metastases. ${ }^{2,9}$ These findings may be attributed to brain metastases increasing tumor angiogenesis, as is the case with GBMs, leading to increased rCBV during the process of growth and invasion. ${ }^{14,32}$ However, depending on the origin and histopathology of the metastases, rCBV values within the tumor tissue may vary over a large range because hypervascular metastases such as renal cell carcinoma and melanoma may have a markedly high rCBV compared with less vascularized metastases. ${ }^{38}$

Thus, combined assessment of capillary permeability and anaerobic metabolism or cellular membrane turnover reflecting cellular proliferation using the combined classifiers $\mathrm{PSR}_{\text {max }}-\mathrm{Lac} / \mathrm{Cr}$ or $\mathrm{PSR}_{\max }-\mathrm{Cho} / \mathrm{Cr}$ was the underlying physiologic and metabolic characteristic that provided the best differential diagnostic performance to discriminate metastases from PCNSLs and GBMs.

Our study may have a few potential limitations, including the small sample size. Furthermore, rCBV measurements using DSC MR imaging in regions of disrupted $\mathrm{BBB}$ with consecutive leakage of contrast agent into the interstitial space may not be entirely accurate. The T1-related signal increase of the contrast agent leakage can partly compensate for the $\mathrm{T} 2{ }^{\star}$-related signal decrease, which may lead to an under- or overestimation of hemodynamic parameters. ${ }^{6,8}$

\section{CONCLUSIONS}

Our results show that perfusion and spectroscopic MR imaging highlighted salient features in lymphomas, glioblastomas, and metastases. Moreover, spectroscopy yielded added value to perfusion using optimal combined classifiers of these modalities, significantly increasing the differential diagnostic performance with a high accuracy for these common brain tumors in daily clinical practice.

\section{REFERENCES}

1. Wang S, Kim S, Chawla S, et al. Differentiation between glioblastomas, solitary brain metastases, and primary cerebral lymphomas using diffusion tensor and dynamic susceptibility contrast-enhanced MR imaging. AJNR Am J Neuroradiol 2011;32:507-14 CrossRef Medline

2. Law M, Yang S, Wang H, et al. Glioma grading: sensitivity, specificity, and predictive values of perfusion MR imaging and proton MR spectroscopic imaging compared with conventional MR imaging. AJNR Am J Neuroradiol 2003;24:1989-98 Medline

3. Mangla R, Kolar B, Zhu T, et al. Percentage signal recovery derived from MR dynamic susceptibility contrast imaging is useful to differentiate common enhancing malignant lesions of the brain. AJNR Am J Neuroradiol 2011;32:1004-10 CrossRef Medline

4. Surov A, Meyer HJ, Wienke A. Correlation between apparent diffusion coefficient (ADC) and cellularity is different in several tumors: a meta-analysis. Oncotarget 2017;8:59492-99 CrossRef Medline

5. Shin JH, Lee HK, Kwun BD, et al. Using relative cerebral blood flow and volume to evaluate the histopathologic grade of cerebral gliomas: preliminary results. AJR Am J Roentgenol 2002;179:783-89 CrossRef Medline

6. Aronen HJ, Gazit IE, Louis DN, et al. Cerebral blood volume maps of gliomas: comparison with tumor grade and histologic findings. $\mathrm{Ra}$ diology 1994;191:41-51 CrossRef Medline

7. Cha S, Lupo JM, Chen MH, et al. Differentiation of glioblastoma multiforme and single brain metastasis by peak height and percentage of signal intensity recovery derived from dynamic susceptibility-weighted contrast-enhanced perfusion MR imaging. AJNR Am J Neuroradiol 2007;28:1078-84 CrossRef Medline

8. Xing Z, You RX, Li J, et al. Differentiation of primary central nervous system lymphomas from high-grade gliomas by $\mathrm{rCBV}$ and percentage of signal intensity recovery derived from dynamic susceptibility-weighted contrast-enhanced perfusion MR imaging. Clin Neuroradiol 2014;24:329-36 CrossRef Medline

9. Stefan D, Cesare FD, Andrasescu A, et al. Quantitation of magnetic resonance spectroscopy signals: the jMRUI software package. $\mathrm{Mea}$ surement Science and Technology 2009;20:104035 CrossRef

10. Vanhamme L, van den Boogaart A, Van Huffel S. Improved method for accurate and efficient quantification of MRS data with use of prior knowledge. J Magn Reson 1997;129:3-43 Medline

11. Smith SM. Fast robust automated brain extraction. Hum Brain Mapp 2002;17:143-55 CrossRef Medline

12. Haker S, Wells WM 3rd, Warfield SK, et al. Combining classifiers using their receiver operating characteristics and maximum likelihood estimation. Med Image Comput Comput Assist Interv 2005;8 (Pt 1):506-14 Medline

13. Liao W, Liu Y, Wang X, et al. Differentiation of primary central nervous system lymphoma and high-grade glioma with dynamic susceptibility contrast-enhanced perfusion magnetic resonance imaging. Acta Radiol 2009;50:217-25 CrossRef Medline

14. Paulson ES, Schmainda KM. Comparison of dynamic susceptibilityweighted contrast-enhanced MR methods: recommendations for measuring relative cerebral blood volume in brain tumors. Radiology 2008;249:601-13 CrossRef Medline

15. Levin JM, Wald LL, Kaufman MJ, et al. T1 effects in sequential dynamic susceptibility contrast experiments. J Magn Reson 1998;130: 292-95 CrossRef Medline

16. Runge VM, Kirsch JE, Wells JW, et al. Repeat cerebral blood volume assessment with first-pass MR imaging. J Magn Reson Imaging 1994; 4:457-61 CrossRef Medline

17. Kleihues P, Burger PC, Scheithauer BW. Histological Typing of Tumours of the Central Nervous System. Berlin: Springer; 1993

18. Hakyemez B, Erdogan C, Bolca N, et al. Evaluation of different cerebral mass lesions by perfusion-weighted MR imaging. JMagn Reson Imaging 2006;24:817-24 CrossRef Medline

19. Toh $\mathrm{CH}$, Wei KC, Chang $\mathrm{CN}$, et al. Differentiation of primary central nervous system lymphomas and glioblastomas: comparisons of diagnostic performance of dynamic susceptibility contrast-enhanced perfusion MR imaging without and with contrast-leakage correction. AJNR Am J Neuroradiol 2013;34:1145-49 CrossRef Medline

20. Hardee ME, Zagzag D. Mechanisms of glioma-associated neovascularization. Am J Pathol 2012;181:1126-41 CrossRef Medline

21. Fellows GA, Wright AJ, Sibtain NA, et al. Combined use of neuroradiology and 1H-MR spectroscopy may provide an intervention limiting diagnosis of glioblastoma multiforme. J Magn Reson Imaging 2010;32:1038-44 CrossRef Medline

22. Guillevin R, Menuel C, Abud L, et al. Proton MR spectroscopy in predicting the increase of perfusion MR imaging for WHO grade II gliomas. J Magn Reson Imaging 2012;35:543-50 CrossRef Medline

23. Harting I, Hartmann M, Jost G, et al. Differentiating primary central nervous system lymphoma from glioma in humans using localised proton magnetic resonance spectroscopy. Neurosci Lett 2003;342: 163-66 CrossRef Medline

24. Howe FA, Barton SJ, Cudlip SA, et al. Metabolic profiles of human brain tumors using quantitative in vivo $1 \mathrm{H}$ magnetic resonance spectroscopy. Magn Reson Med 2003;49:223-32 CrossRef Medline

25. Tang YZ, Booth TC, Bhogal P, et al. Imaging of primary central nervous system lymphoma. Clin Radiol 2011;66:768-77 CrossRef Medline 
26. Long DM. Capillary ultrastructure in human metastatic brain tumors. J Neurosurg 1979;51:53-58 CrossRef Medline

27. Roberts HC, Roberts TP, Bollen AW, et al. Correlation of microvascular permeability derived from dynamic contrast-enhanced MR imaging with histologic grade and tumor labeling index: a study in human brain tumors. Acad Radiol 2001;8:384-91 CrossRef Medline

28. Matsumura A, Isobe $T$, Takano $S$, et al. Non-invasive quantification of lactate by proton MR spectroscopy and its clinical applications. Clin Neurol Neurosurg 2005;107:379-84 CrossRef Medline

29. Bertholdo D, Watcharakorn A, Castillo M. Brain proton magnetic resonance spectroscopy: introduction and overview. Neuroimaging Clin N Am 2013;23:359-80 CrossRef Medline

30. Chawla S, Zhang Y, Wang S, et al. Proton magnetic resonance spectroscopy in differentiating glioblastomas from primary cerebral lymphomas and brain metastases. J Comput Assist Tomogr 2010;34: 836-41 CrossRef Medline

31. Al-Okaili RN, Krejza J, Wang S, et al. Advanced MR imaging techniques in the diagnosis of intraaxial brain tumors in adults. Radiographics 2006;26(Suppl 1):S173-89 CrossRef Medline

32. Opstad KS, Griffiths JR, Bell BA, et al. Apparent T(2) relaxation times of lipid and macromolecules: a study of high-grade tumor spectra. J Magn Reson Imaging 2008;27:178 - 84 CrossRef Medline

33. Opstad KS, Bell BA, Griffiths JR, et al. An investigation of human brain tumour lipids by high-resolution magic angle spinning 1H MRS and histological analysis. NMR Biomed 2008;21:677-85 CrossRef Medline

34. Sjøbakk TE, Vettukattil R, Gulati M, et al. Metabolic profiles of brain metastases. Int J Mol Sci 2013;14:2104-18 CrossRef Medline

35. Delikatny EJ, Chawla S, Leung DJ, et al. MR-visible lipids and the tumor microenvironment. NMR Biomed 2011;24:592-611 CrossRef Medline

36. Righi V, Mucci A, Schenetti L, et al. Identification of mobile lipids in human cancer tissues by ex vivo diffusion edited HR-MAS MRS. Oncol Rep 2009;22:1493-96 Medline

37. Server A, Josefsen R, Kulle B, et al. Proton magnetic resonance spectroscopy in the distinction of high-grade cerebral gliomas from single metastatic brain tumors. Acta Radiol 2010;51:316-25 CrossRef Medline

38. Faehndrich J, Weidauer S, Pilatus U, et al. Neuroradiological viewpoint on the diagnostics of space-occupying brain lesions. Clin Neuroradiol 2011;21:123-39 CrossRef Medline 